\title{
Mechanical analysis of infant carrying in hominoids
}

\author{
Lia Q. Amaral
}

Received: 22 February 2007 /Revised: 6 September 2007 / Accepted: 22 October 2007 /Published online: 21 November 2007

(C) Springer-Verlag 2007

\begin{abstract}
In all higher nonhuman primates, species survival depends upon safe carrying of infants clinging to body hair of adults. In this work, measurements of mechanical properties of ape hair (gibbon, orangutan, and gorilla) are presented, focusing on constraints for safe infant carrying. Results of hair tensile properties are shown to be speciesdependent. Analysis of the mechanics of the mounting position, typical of heavier infant carrying among African apes, shows that both clinging and friction are necessary to carry heavy infants. As a consequence, a required relationship between infant weight, hair-hair friction coefficient, and body angle exists. The hair-hair friction coefficient is measured using natural ape skin samples, and dependence on load and humidity is analyzed. Numerical evaluation of the equilibrium constraint is in agreement with the knucklewalking quadruped position of African apes. Bipedality is clearly incompatible with the usual clinging and mounting pattern of infant carrying, requiring a revision of models of hominization in relation to the divergence between apes and hominins. These results suggest that safe carrying of heavy infants justify the emergence of biped form of locomotion. Ways to test this possibility are foreseen here.
\end{abstract}

Keywords Infant carrying · Hair strength · Clinging · Friction $\cdot$ Bipedalism $\cdot$ Reduction of body hairs

L. Q. Amaral $(\bowtie)$

Department of Applied Physics, Institute of Physics, University of São Paulo, P.O. Box 66318, CEP 05315-970 São Paulo, São Paulo, Brazil e-mail: amaral@if.usp.br

\section{Introduction}

All higher primates (except humans) carry their young clinging to their fur from birth (Jolly 1972). The correlation between infant carrying and the form of locomotion of adult primates is clear, but no detailed study has focused on the mechanics of the problem. Among nonhuman primates, there is a change in the carrying pattern of infants by adults (mostly by the mother) as the infant grows (Jolly 1972; de Vore 1965; Goodall 1967; MacKinnon 1974; Fossey 1979, 1983; Tuttle and Watts 1985). Newborns are carried clinging in close ventro-ventral contact, often with additional support from the mother (Hoff et al. 1983). Change to infant support over the adult body (dorsal or lumbar clinging) occurs some months later for all nonhuman higher primates and extends for years in apes. It seems clear that safety in infant carrying imposes limits on the weight of infants.

This work deals with the problem of infant carrying in Hominoids, characterized by increasing body size. Accepted hominoid phylogeny places the branch to the lesser ape gibbon as the oldest. It is followed by the great apes, with the older branch to the arboreal orangutan and the branch originating terrestrial gorilla and chimpanzee coming later, followed by biped hominids (Yunis and Prakash 1982; Ruvolo et al. 1994; Lockwood et al. 2004). Mechanical analyses of constraints for safe infant carrying in the usual primate pattern are reported. Measurements of tensile and friction properties of hominoid hairs are presented and conditions for mechanical equilibrium determined. The usual pattern of primate carrying of heavy infants is shown to be incompatible with bipedalism. This brings a new perspective to the hominization process and to the basic changes at the divergence between apes and hominins. 


\section{Materials and methods}

\section{Samples}

For the experimental study of the mechanical properties of ape hairs, three pieces of skin with hairs were obtained from frozen limbs of dead animals at the Laboratoire d'Anatomie Comparée, Muséum National d'Histoire Naturelle, Paris, France (one gibbon, one orangutan, and one gorilla-chimpanzee was not available). Due to difficulty in obtaining animal skins, the samples came from three individual animals, one piece of about $200 \mathrm{~cm}^{2}$ from each. The three pieces of skin were transported at room temperature immersed in formaldehyde and were treated afterwards by a taxidermist. After the skin was fixed, it became leather, and chemicals were washed out from the hairs, which looked natural. The effect of formaldehyde on hair has been tested in human head hair; results are presented in the Appendix, and evidence is that no significant change occurs in the relevant tensile parameters.

The skin and hair from the different animals studied here are easily recognized by appearance and texture and are species-characteristic. Gibbon hairs are smooth and silky, while orangutan hairs are harsh, hard and very long; gorilla hair has an intermediate appearance. Estimated values of skin thickness and hair length are given in Table 1, with other parameters obtained.

The three skins provided several samples of different areas with the skin side fixed on pieces of wood, leaving the hairs exposed. The use of taxidermized specimens is the only possibility for simulating the natural situation for friction coefficient measurements. The basic data by Schultz (1931) on hair density of primates were also obtained from embalmed animals.
Methods

Several sectioned ape hair cross-sections were examined under a compound microscope (magnification up to 360), and external appearance as well as hair diameters were measured with a Zeiss Axiovert 200 microscope (several positions along the hair length). Ten single hairs of each of the three animals were cut and analyzed on an automated Instron Tensile Tester, at the standard $65 \pm 2 \%$ relative humidity of the air (RH), $20 \pm 2{ }^{\circ} \mathrm{C}$ and speed $2.6 \mathrm{~mm} / \mathrm{min}$. The hair was fixed on one end to a fixed grip and on the other extremity to a movable grip, connected to a pulling force. The defined distance between grips $\left(L_{\mathrm{o}}\right)$ is between 20 and $50 \mathrm{~mm}$. Curves of force (given in Newton, MKS unit) versus longitudinal elongation $L(\mathrm{~mm})$ were obtained; the strain, or relative elongation, is given by $\left(L-L_{\mathrm{o}}\right) / L_{\mathrm{o}}$. Single human head hair (with and without immersion in formaldehyde) was also analyzed as a standard, as a comparison was possible with previous data for human hairs (Robbins 1994; Nikiforidis et al. 1993; Franbourg et al. 2003). Twenty single hairs from each of the three animals, still on the skins, were also analyzed and pulled until detachment from the skin occurred.

For friction measurements, the wooden pieces with the larger areas from each animal were fixed to a movable inclined plane. The other pieces (with areas in the interval $16-76 \mathrm{~cm}^{2}$ ) were used in measurements of the static friction coefficient $\mu$ (hairs against hairs) using variable weights attached to the wood face. The angle of the inclined plane was carefully changed until slipping started at its critical value, and the process was repeated several times to check reproducibility. The $\mathrm{RH}$ and the room temperature (20$26^{\circ} \mathrm{C}$ ) were measured and controlled with a dehumidifier and an air-conditioning system.

Table 1 Parameter values for apes

\begin{tabular}{llll}
\hline & Gorilla & Gibbon & Orangutan \\
\hline Skin thickness $(\mathrm{mm})$ & $\sim 1.5$ & $\sim 0.6$ & $\sim 2.3$ \\
Hair Length $(\mathrm{cm})$ & $\sim 6$ & $\sim 4$ & $\sim 10$ \\
$\varnothing(\mu \mathrm{m})$ & $66 \pm 2(21 \%)$ & $52 \pm 2(19 \%)$ & $120 \pm 4(24 \%)$ \\
$\alpha(\mathrm{N})$ & $17 \pm 2(25 \%)$ & $6.0 \pm 0.6(33 \%)$ & $32 \pm 1(13 \%)$ \\
$F_{\mathrm{E}}(\mathrm{N})$ & $0.38 \pm 0.03(30 \%)$ & $0.18 \pm 0.02(36 \%)$ & $0.49 \pm 0.02(12 \%)$ \\
$F_{\mathrm{r}}(\mathrm{N})$ & $0.67 \pm 0.09(39 \%)$ & $0.29 \pm 0.04(47 \%)$ & $0.79 \pm 0.04(17 \%)$ \\
$S_{\mathrm{r}}$ & $0.26 \pm 0.05(56 \%)$ & $0.28 \pm 0.05(52 \%)$ & $0.07 \pm 0.01(51 \%)$ \\
$\mathrm{E}(\mathrm{GPa})$ & $5.0 \pm 0.6(42 \%)$ & $2.8 \pm 0.3(43 \%)$ & $2.8 \pm 0.2(36 \%)$ \\
\hline
\end{tabular}

Skin thickness and hair length (estimated), hair diameter $\varnothing$, and results obtained from the $n=10$ tensile curves for each ape hair. The regression coefficient $\alpha$ and the elastic limit $F_{\mathrm{E}}$ are measured in the initial linear Hookean part of the curve, while the force $F_{\mathrm{r}}$ and strain $S_{\mathrm{r}}$ at rupture are measured at the end of the curve. Mean values are given with their standard errors. The Young modulus $E$ is given by $\alpha$ divided by the hair crosssectional area (with error propagation). The variation coefficient ( $\mathrm{CV}=100 \times$ standard deviation/average value) is given in parenthesis. 


\section{Results}

\section{Tensile measurements}

Mechanical studies of animal fibers have been extensively performed over the last decades because of interest in textile production (mainly wool, see Feughelman 1997) as well as cosmetics (human head hair, see Robbins 1994). Animal hair is composed of three parts: an external thin cuticle (protective covering with a scale structure from root to tip), a thicker cortex with fibrous proteins, and a central porous medulla, which may be absent in finer hair. Ape hair viewed under an optical microscope are similar in structure to wool and human head hair, as in Fig. 1, where the external appearance with the cuticle scale structure is shown in Fig. 1a and the cross-section inner structure in Fig. $1 b$.

Mechanical properties of natural fibers are defined by the cortex of the fiber and are due to the molecular structure of keratin, which constitutes the cortex, as determined many years ago (Astbury and Street 1931; Astbury and Woods 1933). Extensive work during decades evidenced the dependence of mechanical properties essentially on the cross-sectional area of the fiber, thus enabling basic research to be conducted on relatively few single fibers, eliminating the need for statistical methods on a large number of samples (Feughelman 1997).

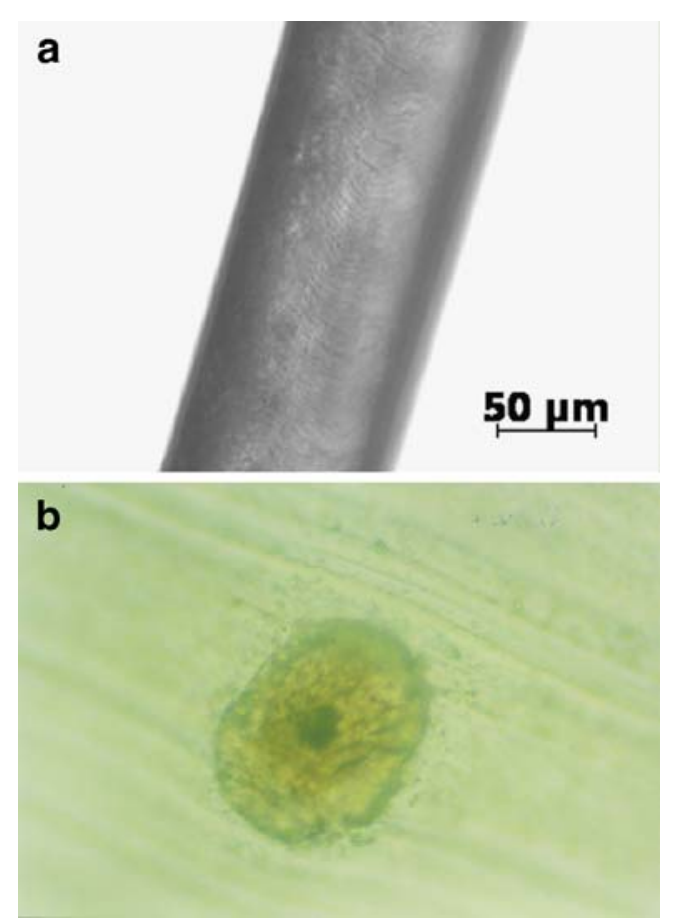

Fig. 1 Micrographs showing orangutan hair, whose light color allows better visualization; a external appearance with typical cuticle scale structure, b transversal cross section with external cuticle, thicker cortex, and inner medulla
Tensile measurements give curves of force against strain (relative elongation) for a single hair fiber. Force has an important meaning for this study; stress is obtained dividing the force by the cross-sectional area of the fiber. A typical result with standard human head hair is shown in Fig. 2a and is similar to earlier data on human hair (Robbins 1994; Nikiforidis et al. 1993; Franbourg et al. 2003). The curve displays an initial elastic (Hookean) linear region, followed by a plastic yield region and a postyield region before breakage. Transition from the elastic to the plastic region corresponds to a first-order transition of the alpha-keratin molecule from the coiled helices to the stretched betakeratin form (Bendit 1957), and the plastic region is strongly influenced by the water content (Feughelman 1997).

The linear region extends up to $1 \%$ strain, a result well established for helical alpha-keratins of all animal hairs: the cortex composite has mechanical properties of a single material (Feughelman 1997). The value of the force at the Hookean elastic limit (called $F_{\mathrm{E}}$ in this paper) corresponds to the limiting force for full recovery of the fiber after being relaxed. The regression coefficient of the linear region (called $\alpha$ in this paper) of the force versus strain curve divided by the cross-sectional area of the fiber gives the Young modulus of elasticity $(E)$ of the fiber (dimension of pressure unit, $\mathrm{GPa}=10^{9} \mathrm{~N} / \mathrm{m}^{2}$ ). The values $\alpha$ and $F_{\mathrm{E}}$ are determined expanding the initial elastic part of the curve (which does not, in general, start precisely at zero), as seen in the insert in Fig. 2a. Further analysis of human head hair is given in the Appendix.

Apparently, no tensile studies on ape hair have been reported. Curves obtained with $n=10$ ape hairs of each of the three apes (one gorilla, one gibbon, and one orangutan) are shown in Fig. 2b-d. The curves display all the classical regions of animal hair. Human hair has a larger postyield region and a larger strain at breakage, related to the usual human hair treatment (frequent washing and combing). Maximum stretching is very different in the wet $(50 \%$ strain) and dry (10\% strain) states and may be influenced also by hair conditioners (Robbins 1994; Colombera and Joekes 2004).

The initial linear region is well defined in all hair curves, and its variability among different hairs of the same ape is due to differences in hair cross-sectional area. The physical parameters $\alpha$ and $F_{\mathrm{E}}$ of each of the ape curves were obtained with the procedure shown in the insert of Fig. 2a. Values of force $\left(F_{\mathrm{r}}\right)$ and strain $\left(S_{\mathrm{r}}\right)$ at rupture were measured directly at the end of the curves.

Table 1 gives statistical averages of parameters obtained from tensile curves $\left(\alpha, F_{\mathrm{E}}, F_{\mathrm{r}}, S_{\mathrm{r}}\right)$ and measured hair diameter $\varnothing$ for each species, together with calculated Young modulus of elasticity $E$, with respective errors. The average values for $F_{\mathrm{E}}$ are $60 \%( \pm 2 \%)$ of $F_{\mathrm{r}}$ for the hairs of the three ape species. Hair diameters $\varnothing$ in Table 1 are 
Fig. 2 Measured force $\times$ strain (relative elongation) curves for hominoid a human, b gorilla, c gibbon, and $\mathbf{d}$ orangutan hairs. In $\mathbf{a}$ are stressed the initial Hookean linear elastic region $(E)$, the plastic plateau $(P)$, the post-yield region $(P Y)$, and the breaking threshold $(B)$. The different curves in $\mathbf{b}, \mathbf{c}$, and d correspond to ten hairs of each individual ape. The inset in $\mathbf{a}$ is a magnification of the elastic linear region, showing the slope $\alpha$ and the force at the elastic limit $F_{\mathrm{E}}$

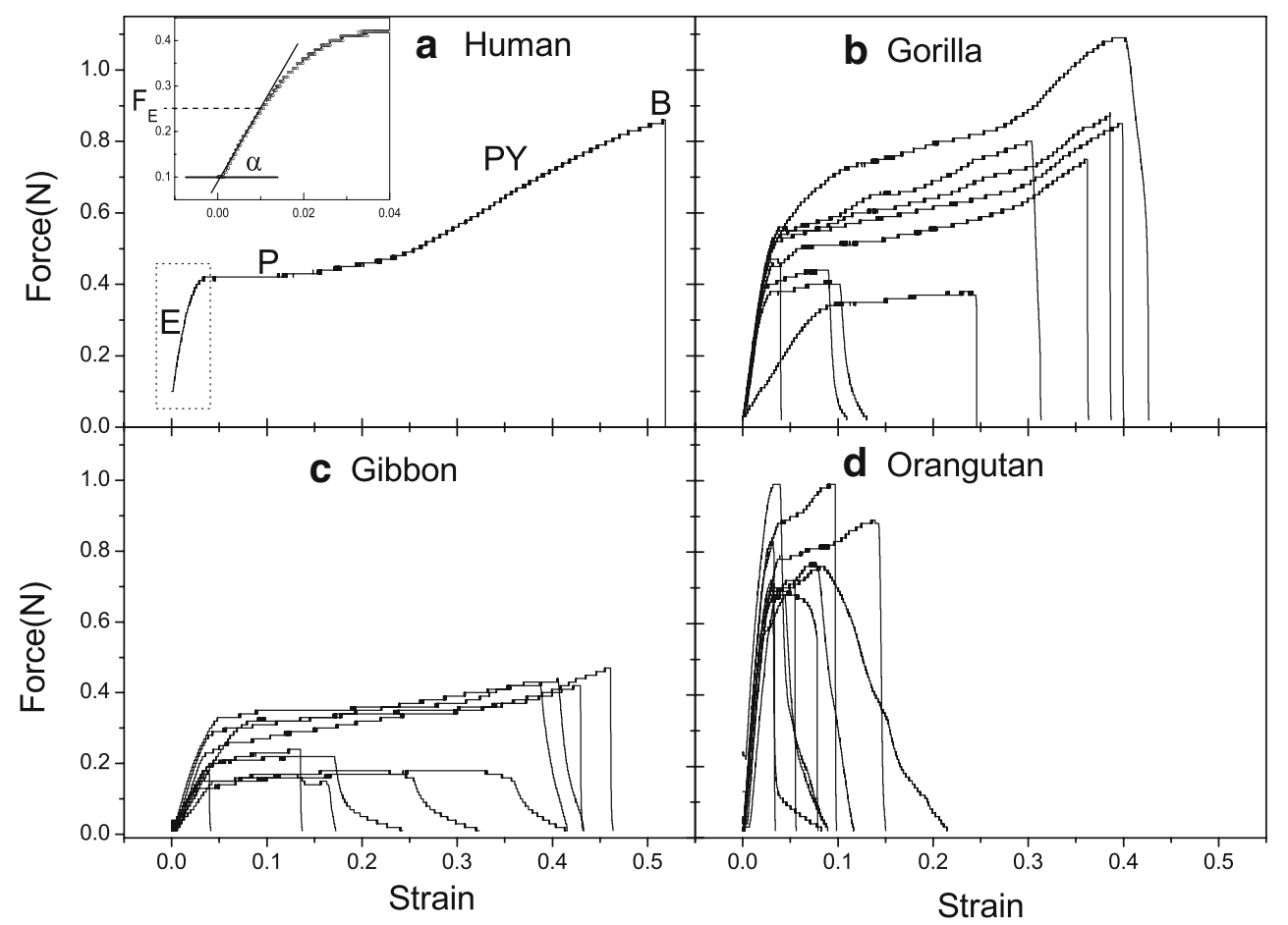

averages over several positions measured along ten single hairs of each ape ( 50 measurements for each ape). Oneway analysis of variance (ANOVA) test for these $\varnothing$ measurements evidenced significant difference between apes $\left(F_{2,155}=177 ; p<0.0001\right)$.

All tensile parameter values for gorilla hairs, with the only exception being the Young modulus $E$, are intermediate between gibbon and orangutan hairs. Table 2 gives statistical tests for these four parameters: significant differences result from two-population $t$ tests and one-way ANOVA test.

Hominoid species differ significantly regarding hair strength, which increases from gibbon to gorilla and to orangutan. However, the Young modulus has a different behavior: $E$ values for gibbon and orangutan are equal
(2.8 Gpa), but gorilla has a higher value (5.0 Gpa). The value for human head hair from literature (Robbins 1994; Nikiforidis et al. 1993) is $3.8 \mathrm{Gpa}$, intermediate between gorilla and orangutan hair. Human head hairs measured in this paper are within reported values, as detailed in the Appendix.

Results obtained indicate that the force strength and average thickness of hominoid hairs are species-dependent and may be evolutionary meaningful. Data for human hair in the Appendix give further indication of species dependence. Such direction of research, together with definition of the Young modulus in terms of the inner hair structure, have clear interest but outside the scope of this work.

For infant carrying, the really decisive parameter is $F_{\mathrm{E}}$, representative of the upper limit of the force that a single

Table 2 Statistics for tensile parameters of ape hairs

\begin{tabular}{|c|c|c|c|c|c|}
\hline & & $F_{\mathrm{E}}$ & $\alpha$ & $F_{\mathrm{r}}$ & $S_{\mathrm{r}}$ \\
\hline \multirow[t]{2}{*}{ Gibbon $\times$ Orangutan } & $t_{10}$ & 11 & 18 & 8.4 & 4.4 \\
\hline & $p$ & $<0.0001$ & $<0.0001$ & $<0.0001$ & 0.0003 \\
\hline \multirow[t]{2}{*}{ Gorilla $\times$ Gibbon } & $t_{10}$ & 5.3 & 6.2 & 4.1 & 0.32 \\
\hline & $p$ & $<0.0001$ & $<0.0001$ & 0.0008 & 0.75 \\
\hline \multirow[t]{2}{*}{ Gorilla $\times$ Orangutan } & $t_{10}$ & 3.1 & 7.6 & 1.3 & 4.0 \\
\hline & $p$ & 0.007 & $<0.0001$ & 0.2 & 0.0009 \\
\hline \multirow[t]{2}{*}{ ANOVA } & $F_{2,27}$ & 44 & 119 & 20 & 9.3 \\
\hline & $p$ & $<0.0001$ & $<0.0001$ & $<0.0001$ & 0.0009 \\
\hline
\end{tabular}

Results from statistical tests for the $n=10$ measured values of the four parameters obtained from the force $\times$ strain curves of hairs of the three apes. Two-population $t$-test ( $t_{10}$ values and corresponding probability $p$ ) are given for each parameter and each compared population in the upper lines. ANOVA test of the four parameters in the three ape populations and corresponding probability $p$ in the lower line 
hair can safely withstand, because it is the limit for recovery after relaxing.

It is convenient to express $F_{\mathrm{E}}$ in kilogram-force (and gram-force), corresponding to the force weight of a given mass at the earth surface. This unit is often still used for load (instead of $N=0.10198 \mathrm{kgf}$ ) because it makes the mass value of the weight clear. The important values $F_{\mathrm{E}}$ are then $18 \pm 2$ gf for gibbon, $39 \pm 3$ gf for gorilla, and $50 \pm 2$ gf for orangutan. The value for human head hair is $29 \pm 2$ gf (see Appendix).

The force to pull hairs from the skin samples of the three ape species has also been measured and may be considered as an upper limit, in view of changes in skin due to taxidermy. They are typically near the elastic limit and lower than rupture value, as occurs with human head hair in the scalp (Robbins 1994), giving a further reason to focus on the $F_{\mathrm{E}}$ values as upper limits for safe load carrying.

A robust conclusion can be thus extracted. It is clear that to withstand the clinging infant weight, a large number of hairs held together is required. Results obtained evidence that bunches of about 100 hairs are necessary to carry infants weighing a few kilogram-force. Infant hands (and feet) can possibly grasp a bunch of hairs available in some square centimeters of skin so that safety in the usual pattern of nonhuman primate infant carrying critically depends on the density of hairs.

\section{Infant carrying}

Values of hair density for nonhuman primates have been obtained in the extensive work of Schultz (1931, 1968); values are not gender-dependent but vary with body region: density is highest in the vertex and smaller on the ventral than on the dorsal side of the trunk. Densities are generally compatible with the carrying requirement defined above, but the great apes have the lowest hair density among primates. Hair density values on the back of primates vary, from over 1,000 hairs $/ \mathrm{cm}^{2}$ for smaller species (monkeys and gibbons), down to about 100 hairs $/ \mathrm{cm}^{2}$ for great apes. The problem of safely carrying heavy infants requires thus particular consideration among the great apes.

Analysis of hair density data (Schultz 1931) in many anthropoid primate taxa yield (Schwartz and Rosenblum 1981) a negative allometry of relative hair density ( $r h d=$ hair density/total surface area). This decrease in rhd with increase in primate body mass has been correlated to thermal constraints imposed by the decreasing ratios of surface area to body volume. However, chimpanzees apparently have a lower rhd than would be expected for their body volume (see Fig. 1 of Schwartz and Rosenblum 1981), with rhd similar to that of larger gorillas.

The bunch of hairs available for clinging depends also on hair length and infant hand size. Accepting that about
$3 \mathrm{~cm}$ are necessary to roll hairs on the infant fingers, the excess in length defines the area available for clinging. Clinging area per limb may be thus estimated to be 3,10 , and $50 \mathrm{~cm}^{2}$ for, respectively, gibbon, gorilla, and orangutan. In a dynamic situation, the load should be supported instantaneously by a single limb.

The infant-carrying method of the arboreal Asian apes (gibbon and orangutan) is different from that of the terrestrial African apes (gorilla and chimpanzee). The arboreal apes carry their young over one side of the mothers' pelvis, not on the back (de Vore 1965). In accordance with the small size (7 kgf when adults), the lesser ape gibbon has a high hair density, over 1,000 hairs $/ \mathrm{cm}^{2}$, more than ten times the density of great apes (Schultz 1931, 1968). This ensures safety for carrying their light young in their acrobatic arboreal life. The arboreal but heavier orangutans (adult females weigh around $50 \mathrm{kgf}$ and males $90 \mathrm{kgf}$ ) compensate the lower hair density by longer, thicker, and stronger hairs and thicker skin. Orangutan infants live with their mothers for about 7 years.

The gorilla is about $50 \%$ heavier than orangutan, but instead of even greater hair thickness and length, the solution for terrestrial apes is the mounting position for heavier infant support. Orangutan hair and skin are, in this paper, estimated to be able to withstand about seven times more weight than gorilla hair and skin. Gorillas are the heaviest primates and have very few chest hairs (Schultz 1931, 1968). Adult gorilla is much heavier than adult human, but their newborns have only half the weight of human babies. In the first 1 or 2 months, the infant gorilla is supported manually by its mother as she walks tripedally or bipedally (Jolly 1972; de Vore 1965; Tuttle and Watts 1985; Doran 1997). The same occurs for chimpanzee babies, unable to support their own weight by clinging prior to 2 months of age (Plooij 1984). Change from quadruped to triped or biped motion occurs systematically among all great apes when infant safety requires manual support (Jolly 1972; de Vore 1965; Tuttle and Watts 1985). Slow and careful locomotion of female chimpanzees while carrying young infants has been reported (Goodall 1967). Two primary causes of mortality have been found among infant chimpanzees: inadequacy of the mother-infant bond and injuries caused by falling from the mother (Goodall 1967). It is clear that infant carrying is crucial and depends on not trivial behavior among African great apes.

\section{Weight limit for clinging in African apes}

The gorilla $F_{\mathrm{E}}$ value, combined with hair density and length, indicates that load up to $40 \mathrm{kgf}$ could be supported by clinging. However, the weight limit for clinging depends also on several other variables. Increase in hair pull-out or break in the dynamic situation, capacity of the infant to 
withstand its own weight and to grasp hairs without sliding, and skin capacity to withstand the load without rupture are all variables that may decrease the infant weight limit for clinging.

A bunch of hairs on about $1 \mathrm{~cm}^{2}( \pm 10 \%)$ of gorilla skin under load was observed, still on the skin, to simulate the real load effect in the actual clinging situation. Counting hairs in this bunch gave 140 hairs $( \pm 10 \%)$, in agreement with the value reported by Schultz (1968) for the back side of the trunk of gorillas (145 hairs $\left./ \mathrm{cm}^{2}\right)$, evidencing that there are no marked differences between the limb sample and the back-trunk hairs. This bunch of hairs supported $1 \mathrm{~kg}$ without problems, even with some pendulum movement. With $2 \mathrm{~kg}$, several problems became evident. Besides eventual detachment of single hairs from the skin during movement, the hair bunch started to slip from the double-face adhesive tape used to attach it to the load support. It was necessary to make a new attachment system; the hair bunch was rolled several times around the weight support. Even so, loads of $2 \mathrm{~kg}$ and more could be observed only with the rolled hairs attached by super glue (ester cyanoacrylate) to the weight support. This indicates a possible problem also with the clinging capacity of infant fingers, and slippage may occur. Also, the skin itself began to deform and detach from the wood support, requiring a much stronger attachment of the skin to the wood base. The bunch of hairs broke at the same hair length for all under a static load of $7 \mathrm{~kg}$, compatible with the values of force at rupture $\left(F_{\mathrm{r}}\right)$ obtained from the tensile measurements of single hairs (Table 1). An upper limit of $1 \mathrm{kgf} / \mathrm{cm}^{2}$ for clinging without problems may be deduced from this simple experiment, but the actual limit depends on the clinging capacity of infant fingers and on the mother's skin resistance. The effective weight limit for clinging must be obtained from careful observation of live apes.

Fossey (1979) describes physical and behavioral development of gorilla infants: at 4-6 months (weight about $5 \mathrm{kgf}$ ), the gorilla infant travels $60 \%$ of the time in ventral position while with 6-12 months (weight about $8 \mathrm{kgf}$ ), it travels in the dorsal position $80 \%$ of the time. A detailed investigation of the ontogeny of locomotion in the African ape (Doran 1997) revealed that up to 5 months of age, chimpanzees are slightly more precocious than gorillas. However, at 6 months, gorilla locomotor development becomes faster than that of chimpanzees, and surprisingly, much of the interspecific variation in behavior is explained by differences in body size (Doran 1997). Afterward, when the infants are of similar weights (although of widely disparate ages) gorillas and chimpanzees perform very similar locomotion activities (Doran 1997).

It is, in this paper, suggested that the weight level of $5 \mathrm{kgf}$ may be considered the limiting value for safe support over long periods by only ventral clinging, defined probably by a combination of security factors ${ }^{1}$ related to hair strength, infant clinging capacity, and skin resistance to pressure. The limiting clinging pressure on the mother's skin for the African great apes may be thus estimated to be $0.5 \mathrm{kgf} / \mathrm{cm}^{2}$ (a factor of 2 in relation to the simple test made with the skin sample). The skin is also a visco-elastic medium, reflecting properties of a strain-induced alignedcollagen network that can stretch up to $100 \%$ before permanent damage (Silver 1987). The shear modulus for the human dermis ranges from 1.2 to $3.1 \mathrm{MPa}$, while for the human hypodermis, it ranges from 3.1 to $9.7 \mathrm{kPa}$ (Gennisson et al. 2004). The pressure limit $0.5 \mathrm{kgf} / \mathrm{cm}^{2}(\sim 50 \mathrm{kPa})$ could be a limit also for danger to the skin of the mother ape. It is known that one of the important differences between the skin of humans and of African apes is the higher elasticity of the human skin (Montagna 1982).

The locomotor development of the infants must therefore adapt to such an effective weight limit. This does not mean that infants over $5 \mathrm{~kg}$ will fall. Larger infants may travel briefly in ventral position during stressful situations (Fossey 1979) or when the chimpanzee mother swings through trees (Goodall 1967). Usual locomotion of adult African apes is terrestrial quadrupedalism ( $\sim 96 \%$ of the time in gorillas and $\sim 86 \%$ in chimpanzees, from Doran 1997), and safe infant support over longer periods clearly requires the necessity of change to a mounting position, with the infant weight supported by the adult body.

\section{Dorsal clinging position}

In order to analyze the mechanics of the carrying system in the African great apes, it is also necessary to consider, besides the tensile properties of hairs, the hair-hair friction that prevents slipping, particularly for heavier infants in dorsal position. Fig. 3 is a sketch of the mounting position in African apes, showing the angle $\theta$ of the inclined plane where the infant stands. For simplicity in the sketch, the angle $\theta$ coincides with the angle defined by the usual knuckle-walking position of African great apes, but that is not necessary. The important parameter is the inclination of the base where the infant stands clinging; its relation to the average body inclination angle does not need to be taken into account in analysis of the friction effect.

The total infant weight, in the vertical direction, has two components, one in the inclined plane, favoring slipping, and one normal to the inclined plane, responsible for the friction force opposing slipping. Calling $W_{\mathrm{t}}$ the total infant weight carried in mounting position, $W_{\mathrm{c}}$ the effective

\footnotetext{
${ }^{1}$ High-security factors are usual for the use of ropes by firemen and workers in civil construction and cargo transport. A ratio 1:15 between the safe and rupture load is deduced from NFPA 1983 (Standard for Life Safety Rope and System Components, National Fire Protection Association).
} 
weight limit for clinging, and $\mu$ the static friction coefficient (Bowden and Tabor 1956), the equilibrium of forces on the infant requires that force components on the inclined plane direction satisfy the condition:

$\mu W_{\mathrm{t}} \cos \theta+W_{\mathrm{c}}>W_{\mathrm{t}} \sin \theta$

In the absence of clinging $\left(W_{\mathrm{c}}=0\right)$, slipping on the body surface of contact starts for a critical value $\theta_{c}$, given by tan $\theta_{\mathrm{c}}=\mu$. Both friction and clinging are thus essential to hold heavy infants in the mounting position. The requirements for dynamic equilibrium may be greater, so that in fact the mounting position in dynamic movement may be unsafe even when Eq. 1 is satisfied.

It should be stressed that Eq. 1 is a necessary condition for infant survival and therefore also for species survival. It is a very robust requirement of basic mechanics for static equilibrium. The ape hair-hair friction coefficient $\mu$ must be known to analyze the actual ape situation.

\section{Friction measurements}

Apparently, no results for hair-hair friction coefficients exist in the literature; only friction between human hair and other materials are reported (Robbins 1994). The skin samples were used to measure $\mu$ as a function of the supported load $W$ in known conditions of relative air humidity and temperature, in a situation analogous to that depicted in Fig. 3. It was verified that $\mu$ depends on the hair direction, being larger for hairs in the parallel position (as in nature, with the two animals heading in the same direction) than in the antiparallel condition. Systematic measurements have been thus made in the parallel condition.

Figure 4a shows a typical result of $\mu$ as a function of $W$. For hard solid surfaces, $\mu$ is constant and independent of the load $W$ and contact area $A$. This is clearly not the case for hairs, especially at low loads. Long ago, it was shown that a variation of $\mu$ with $W$ occurs for textiles and fibers (Gralen 1952; Makinson 1952), given by the empirical equation:

$\mu=a+b / W$

The parameter $a$ gives the friction coefficient in the limit of high loads. It has been proposed (Gralen 1952) that the parameter $b$ is related to the pressure inside an oil lubricating film on the fiber surface. The thickness of this film decreases due to pressure, which causes changes in the friction mechanism. Hair is a natural fiber (Howell et al. 1959) and behaves in a similar way. In fact, the data could be well-fit with Eq. 2, shown as a solid line in Fig. 4a.

Many curves were analyzed for different values of the contact area $A$ and RH. A strong dependence of $\mu$ on $A$ was observed, probably due to shear among the several hairs in

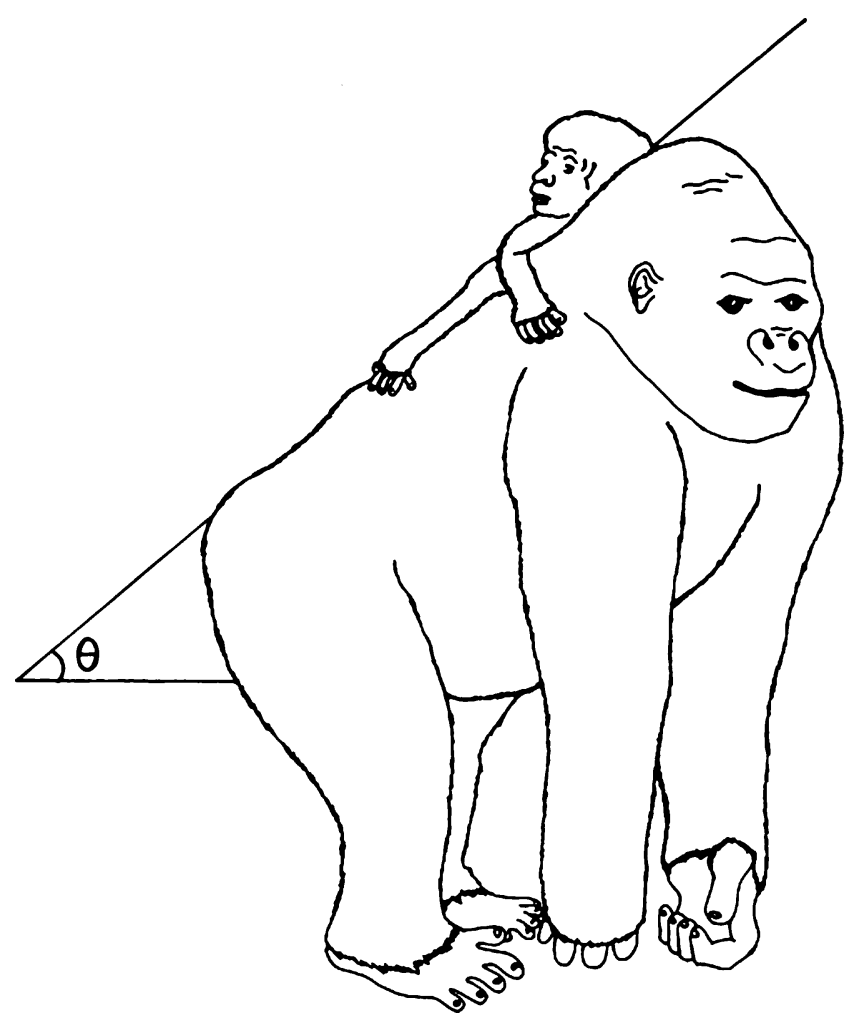

Fig. 3 Sketch of ape carrying infant. For simplicity, in the sketch, the angle $\theta$ of the inclined plane where the infant stands (to which Eq. 1 refers) coincides with the angle defined by the knuckle-walking position of African great apes, which is not necessarily so, as discussed in the text

contact. The fits to the linear equation $\mu \mathrm{W}=a \mathrm{~W}+b$ are more convenient for yielding the parameters $a$ and $b$, as shown in Fig. 4b. Both $a$ and $b$ increase with $A$, but $a$ is less sensitive to $A$, while $b$ is roughly proportional to $A$, so that the term $b / W$ in Eq. 1 is inversely proportional to the pressure $W / A$ exerted on the surfaces in contact.

Results indicate that the friction coefficient might be considered constant for pressures higher than $20 \mathrm{gf} / \mathrm{cm}^{2}$. This pressure corresponds to infant weight around $1 \mathrm{kgf}$, from the relation between body surface area and weight (Schwartz and Rosenblum 1981; Kleiber 1975) and estimating the contact area as one third of the infant surface area.

The average value is $\mu=0.27 \pm 0.03$ for gorilla hairs and $\mu=0.20 \pm 0.02$ for gibbon hairs at $60 \% \mathrm{RH}$, in agreement with the expected null or small difference on friction depending on the fiber diameter (Robbins 1994). Such values are in good agreement with known values for single fibers (Bowden and Tabor 1956; Howell et al. 1959). The orangutan skin was not as perfect and homogeneous as the gibbon and gorilla skins, showing some parts without hairs, and provided only two samples, so dependence on area could not be analyzed. However, experiments of $\mu$ as a function of load gave similar values to those for the other apes. 


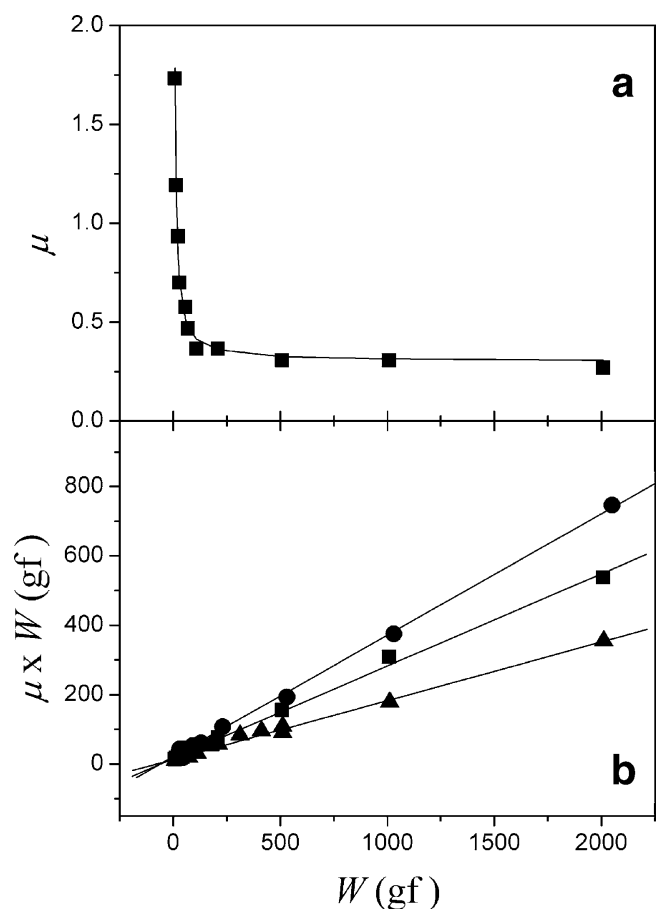

Fig. 4 Results for friction coefficient at $80 \% \mathrm{RH}$. a friction coefficient $\mu$ as a function of $W$ for a gorilla skin with smaller area $\left(16 \mathrm{~cm}^{2}\right)$. The line shows a fit to the data using Eq. 2. b $\mu \times W$ for (circles) gorilla with larger area $\left(76 \mathrm{~cm}^{2}\right)$; (squares) gorilla with smaller area $\left(16 \mathrm{~cm}^{2}\right)$, and (triangles) gibbon with intermediate area $\left(42 \mathrm{~cm}^{2}\right)$. Linear fits to the data are shown in lines

The hair-hair friction coefficient of apes obtained is intermediate between values for friction between human hair and hard rubber with dry $(\mu=0.19)$ and wet $(\mu=0.38)$ surface conditions (Robbins 1994), indicating that results obtained can be considered of general validity. Note that Robbins (1994) mentions the difficulty of testing hair-hair friction, important in hair combing, and that it should be similar to hair-rubber friction.

\section{Equilibrium condition in African apes}

It is possible now to focus the problem on our nearest relatives, the great apes gorilla and chimpanzee, applying Eq. 1 to their mounting condition, in which clinging and friction act together for safe infant carrying.

The effective weight limit for constant ventral clinging, already discussed, is estimated to be $5 \mathrm{kgf}$. As the hair density in the back is higher ( $30 \%$ for chimpanzees), the estimate is $W_{\mathrm{c}} \sim 5-7 \mathrm{kgf}$. The maximum value $W_{\mathrm{t}}$ can be estimated from the weight of infants when they are no longer carried by mothers. This occurs when gorillas are about 2.5 years old and chimpanzees 5 years old, both weighing around $20 \mathrm{kgf}$ (Fossey 1979; Doran 1997).

By inserting such values in Eq. 1, and using the estimated constant friction coefficient $\mu$, it is possible to obtain the maximum inclination of the plane supporting the infant for the safe carrying system in the African great apes. The condition $\theta<29$ to $34^{\circ}$ is obtained.

This basic equilibrium constraint related to friction in the mother-infant contact is satisfied in ape knuckle-walking quadruped position ${ }^{2}$.

It should be stated that it is frequently reported that in the dorsal position, the African ape infant rides high on the mother's neck and lies stretched and prostate (de Vore 1965; Fossey 1979). This position increases the fixed base of the load and places it in a body region with a promontory and a smaller inclination angle $\left(\theta \sim 20^{\circ}\right.$ as estimated ${ }^{2}$ from the same Fig. 7-20 in Schultz 1968). Older infants ride in a jockey position; they do not normally grip hairs with their feet, but press the sides of their ankles against the mother's flank (Goodall 1967). Clearly, among terrestrial great apes, infant safety does not rely only in grasping hairs.

It may be concluded that slipping imposes clear limits on the maximum body angle attained by heavy ape species carrying infants, representing a hindrance against evolution towards bipedality. This might explain the persistence of knuckle-walking among the great African apes.

\section{Effect of humidity on friction}

The two most relevant variables to friction in hairs (Robbins 1994) are the load pressing the two surfaces together, as analyzed previously, and the relative humidity RH. Dependence of $\mu$ on RH was reported for fibers (Howell et al. 1959) and human hair (Robbins 1994), and a similar effect was verified for ape hairs.

Reduction in RH, from 80 to $38 \%$, led to a decrease of gorilla hair $\mu$ by about $30 \%$. The friction coefficient between human hair and hard rubber is reduced by $50 \%$ between the wet and dry states (Robbins 1994).

Friction of gorilla hairs has been also measured in wet conditions (immersion in water, followed by slight shaking of the sample). The result is $\mu=0.35 \pm 0.03$, in good agreement with reported values for human hair and hard rubber in the wet condition. The larger $\mu$ value in wet condition is possibly due to network of hydrogen bonds connecting hairs in contact. The thin outer epicuticle membrane of hair is hydrophobic, but hydrogen bonds may occur through the thicker exocuticle and endocuticle, especially the latter, which swells much more in water (Feughelman 1997, Robbins 1994). Such structures may be accessible to external water through the ratchet scale structure of hair.

\footnotetext{
${ }^{2}$ This assertion comes from analysis of many ape pictures and line drawings of standard lateral views (as Fig. 7-20 in Schultz 1968, from which an average body angle of $26^{\circ}$ is estimated), as no values for average body angles could be obtained from the literature.
} 
It should be concluded that reduction in humidity destroys the delicate balance between friction and clinging (Eq. 1) necessary for survival of infants of heavy ape species. Figure 5 shows the maximum carrying weight $W_{\mathrm{t}}$ obtained from Eq. 1 as a function of the inclination angle $\theta$ for $\mu$ varying from zero up to 0.40 , with $W_{\mathrm{c}}=5 \mathrm{kgf}$.

\section{Discussion}

In this section, the relevance of the above results for models of hominization is discussed. Safe infant carrying is clearly very important for species survival and has to be considered in the discussion of the transition from apes to hominins. The mechanical analysis made in this paper evidenced that habitual bipedalism, with a large $\theta$ angle, is incompatible with the usual primate type of infant carrying. Even if one considers $W_{\mathrm{c}}=10 \mathrm{kgf}$ and $\mu=0.40$, the maximum attainable angle is $\theta \sim 49^{\circ}$, as seen in Fig. 5.

It seems clear that together with bipedality probably also came the characteristic human type of infant carrying in the arms and hands of their mothers, making their locomotion and foraging particularly difficult. Females would not adopt bipedality if they could avoid it, and the species would not adopt bipedalism if females with infants would not engage in it. The transition to bipedality was not trivial.

Etkin (1954) considered the problem of the burden on females "almost continuously carrying a child," just to conclude that the female could not be an effective hunter, turning to a theory of the monogamous family unit. It is clear now that such a scenario could be considered only for Homo

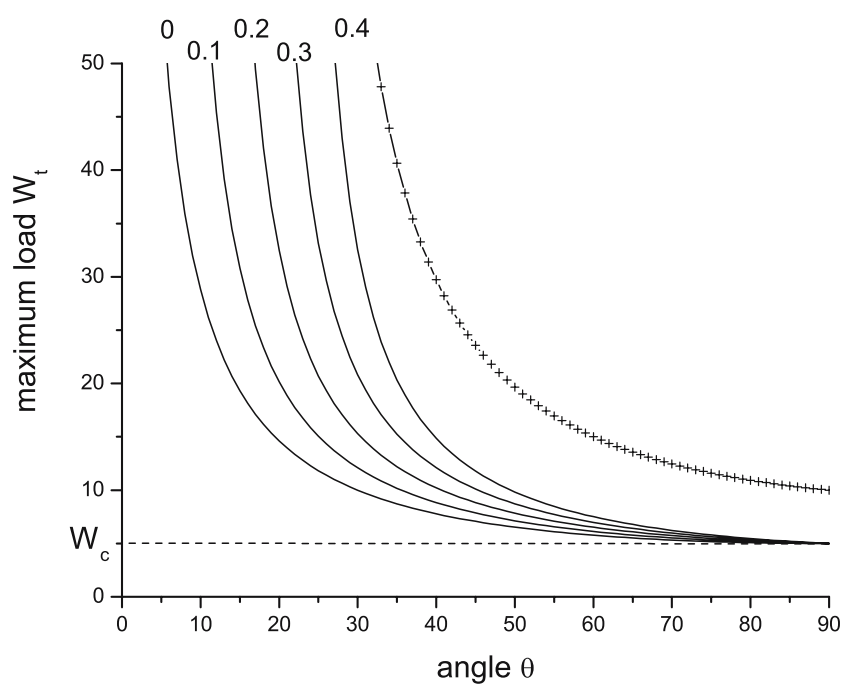

Fig. 5 Curves of total weight $W_{\mathrm{t}}$ supported in dorsal position as function of the inclination angle $\theta$ of the infant base on the mother's trunk, obtained from Eq. 1, for several values of the friction coefficient $\mu\left(0-0.4\right.$, as indicated on top), with $W_{\mathrm{c}}=5 \mathrm{kgf}$ (limit value shown with dashed line). The line with cross is for $W_{\mathrm{c}}=10 \mathrm{kgf}$ and $\mu=0.4$. Vertical position corresponds to $\theta=90^{\circ}$ at a much later period. Iwamoto (1985) recalled examples of facultative bipedalism among monkeys and speculated that the "decisive factor (for habitual bipedalism) may have been some everyday necessity to carry something in both hands". After criticizing proposals in which the "something" is food, Iwamoto suggested that the "something" could be their helpless babies. However, this proposal has been dismissed under the hypothesis that babies became helpless only with increase of brain in Homo.

Now models for bipedalism origin based on "food acquisition and foraging strategies" are considered "likely" but are in fact based on the premises that bipedal locomotion frees the arms and hands and does not have high costs. However, such premises just forget the fundamental fact that bipedalism frees the arms and hands only of males and juveniles, but females, on the contrary, have arms and hands occupied by infant carrying, which represents a high cost for bipedalism, not considered up to now. A review of bipedalism mentions: "infants (from early hominin biped) probably clung to their mothers' hair, as is the case for most other primates" (Richmond et al. 2001).

It seems that the not trivial mechanical constraint of safe carrying of heavy infants has never been considered before.

Current evidence suggests (Richmond et al. 2001; Kingston et al. 1994; Potts 1998) that the key adaptation to bipedalism originated in wooded environments during a drier period in East Africa. The equilibrium condition (Eq. 1) shows that reduction of friction in the dorsal clinging position would most probably lead to a decrease in infant weight and decrease in the body inclination angle $\theta$, corresponding to the solution adopted by quadruped savanna monkeys in the arid savanna. It seems clear that habitual bipedalism would not be reached by continuous increase of the body angle. However, among hominoids, reduction of friction between infant fingers and mother hairs could reduce infant grasping capacity, increasing the period of mother's manual support of newborns and eventually inducing habitual bipedalism.

\section{Concluding remarks and future perspectives}

An important conclusion from this work refers to interest in systematic study of tensile properties of hairs from an evolutionary point of view. The other clear conclusion is that models on locomotion and bipedalism evolution must focus on females carrying infants, who are the target of the strongest selective pressures, and this conclusion is independent of the form of locomotion of hominid ancestors (knuckle-walkers, terrestrial or arboreal quadrupeds, or fulltime arboreal climbers).

To disentangle the divergence between apes and hominins, several directions of research become clear from this 
work. Studies of living apes focused on the mechanical problem of infant carrying, as well as determination of the elastic limits of the ape skin and the limits for grasping capacity of ape infants, which are all essential to identify the critical factor responsible for emergence of bipedalism.

It should be stressed that in a scenario with bipedalism emerging for safe infant carrying, the selective pressure would act particularly on females. This is not a problem, as only genes in the nonrecombinant part of the Y chromosome are not transmitted to both sexes and therefore cannot be associated with species locomotion. The process correlates with increase in the mother-infant bond, characteristic of the human lineage.

Other hypotheses for emergence of bipedality (see Richmond et al. 2001 for a review) do not, in fact, explain why other primates did not follow such line. The many scenarios invoked previously for bipedalism evolution may sound plausible, but are not necessary; neither take into account the problem of infant carrying and can be considered complementary and a consequence of the selective pressure on females carrying infants. The difficulties in locomotion and food gathering for biped females carrying infants may well be at the origin of the necessity of group cooperation, which could initially have been among females, with males in their usual role of primate group protection.

A fundamental question is the possible correlation between bipedalism and reduction of body hairs, the two basic biological modifications of hominins. It is evident that decrease in body hair as the initial modification would bring on bipedality as a necessary consequence (Amaral 1989), through the strong selective pressure of safe infant carrying, when infants could no longer cling to body hairs.

The timing of reduction of body hairs is very controversial, since Darwin's original (1871) emphasis on the issue. Several works indicated a very early beginning of body hair reduction, still in forested environments, based on thermoregulatory requirements (Newman 1970), rhd of Australopithecines (Schwartz and Rosenblum 1981), and comparison of human and ape skin (Montagna 1982, 1985). Bipedality preadaptive to nakedness was suggested later from water-consumption requirements under reduced humidity (Wheeler 1992). However, detailed analysis of thermal loads and water consumption for quadrupeds and bipeds in the furred and naked conditions suggested again that reduction of body hair started in a forested environment becoming drier (Amaral 1996). Savanna monkeys tolerate heat even at high levels of exercise, evidencing that the biological avenue to cope with heat stress in an open environment is to keep a hair covering, increase sweating capacity, and have a variable conductance (patas monkeys, Mahoney 1980, and baboons, Rogers et al. 1992). The only situation in which nakedness is favorable concerns dissipa- tion of heat loads that do not come from sun absorption but from activity in a more closed forest condition at temperatures below that of the body (Amaral 1996). Furthermore, regarding water requirements, advantages for nakedness exist in dry ambients with temperatures below body temperature (Amaral 1996).

Recent genetic work has focused on evolution of black skin as a result of naked unprotected skin under solar stress, estimated to have occurred at least 1.2 mya (Rogers et al. 2004), while clothing is a recent innovation of only about 70,000 years ago (Kittler et al. 2003). However, up to now, there is no date for the beginning of reduction of body hairs. Therefore, localization of the genetic changes responsible for reduction of body hairs in humans is necessary to settle the issue.

The new perspective developed in this paper evidences continuity between physical and behavioral aspects of early hominins and their ancestors. The more differentiated aspects of humans are left to the emergence of Homo, at a much later period.

Acknowledgments Thanks are due to Dr. Christine Berge for the kind supply of the three animal skins, to Dr. Walter A. Neves for the use of the room with controlled humidity, and to both for conversations on human evolution; to the Laboratory of Textiles, Instituto de Pesquisas Tecnológicas (IPT), for the tensile measurements and micrographs of hair cross sections; to Karla Colombera and Dr. Otaviano Helene for useful technical information on analysis of, respectively, tensile parameters and statistics, and to João Carlos Terassi for the drawing in Fig. 3. Discussions with Dr. Karin A. Riske were important to give form to the several versions of the manuscript; I thank her also for micrograph measurements of hair diameter. The experiments comply with the current laws of Brazil.

\section{Appendix}

Effect of formaldehyde on hair

Formaldehyde is known as a cross-linking agent reducing water content and increasing mechanical strength (Gross et al. 1997; Honda et al. 1997). It has dramatic effects on skin but is present in many hair shampoos and lacquer.

In order to test the effect on hair tensile properties, ten single human hairs from two volunteers with long hairs (five from a blond woman and five from a man with brown hair) were each cut in three pieces of about $6 \mathrm{~cm}$. Three hair treatments were performed: one piece remained as control, and two pieces were immersed in formaldehyde $(36 \%$ aqueous solution) for a week; one dried afterwards and the other immersed in baths of distilled water for hours before drying. Each piece of hair was examined under an optical microscope before and after treatment and thickness measured in three positions along its length. The average of such three values was considered in further analysis of 
the five treatment procedures (control plus before and after two treatments). The ten single hairs gave a total of 150 measurements of hair thickness $\varnothing(10 \times 3 \times 5)$.

The treatment had some effect on hair external appearance, as formaldehyde straightened curled hairs, and water restored curls. Only one piece out of ten treated with formaldehyde and not washed afterwards broke into small pieces and revealed under the microscope inner corrosion due to damage to the cuticle; none of the ten pieces washed after treatment showed such damage. One of the smaller pieces of the damaged hair was kept for the tensile study.

Tensile curves of the 30 pieces of human hairs were measured under the same condition as ape hairs. The small piece of the damaged hair showed abnormal tensile curve, which was discarded. Several statistical tests were performed to analyze values of tensile parameters and $\varnothing$.

Hair thickness values with one-way ANOVA test on five sets of hair treatment did not show significant differences for each volunteer $\left(F_{4,20}=0.3 ; p=0.9\right.$ and $F_{4,20}=1.5 ; p=$ 0.2 ). This indicates that hair thickness is not significantly changed by formaldehyde treatment.

It is well known that cross-sectional parameters of human head hair are ethnic determined (Wolfram 2003, Franbourg et al. 2003); its diameter varies generally between $60-80 \mu \mathrm{m}$ and is not defined by gender. Analysis of $\varnothing$ data separated by volunteers revealed very significant differences in mean values $(65 \pm 2$ and $81 \pm 2 \mu \mathrm{m}$, with $\mathrm{CV}$ $11 \%)$ and a two-population $t$ test yield $t_{25}=7 ; p<0.0001$. The two volunteers are therefore good examples of large variability in human hair thickness.

One-way ANOVA test performed on the six sets of data ( 3 hair treatments $\times 2$ volunteers) of the more important tensile parameter $F_{\mathrm{E}}$ indicated no significant differences $\left(F_{5,23}=2 ; p=0.1\right)$, evidencing that $F_{\mathrm{E}}$ is not significantly changed by formaldehyde treatment. Separating data from the two volunteers, mean values are $0.31 \pm 0.02 \mathrm{~N}$ (CV $23 \%$ ), and $0.24 \pm 0.02 \mathrm{~N}$ (CV 30\%). A two-population $t$ test evidenced some difference on $F_{\mathrm{E}}\left(t_{15}=3 ; p=0.013\right)$. Total average value is $F_{\mathrm{E}}=0.28 \pm 0.02 \mathrm{~N}(\mathrm{CV} 29 \%)$.

Values of $\alpha$ for each piece of hair together with the average thickness value for that piece yield the calculated Young modulus $E$ ( 10 pieces of hair $\times 3$ hair treatments). One-way ANOVA test on $E$ values indicated no significant differences between the control and pieces treated with formaldehyde $\left(F_{5,23}=0.9 ; p=0.5\right)$. Separating $E$ data for the two volunteers, average values are $3.1 \pm 0.2 \mathrm{Gpa}$ (CV 32\%) and $2.7 \pm 0.2 \mathrm{Gpa}(\mathrm{CV} 42 \%$ ); however, a two-population $t$ test indicated no significant difference on $E\left(t_{15}=1 ; p=0.3\right)$. The average value over all data yield $E=2.9 \pm 0.2 \mathrm{Gpa}(\mathrm{CV}$ $33 \%)$.

The value in this paper reported for $E$ in human head hairs is within the reported variation for human hairs (Nikiforidis et al. 1993) among thin human hairs $(E=$
$3.8 \mathrm{Gpa}$ for $\varnothing<70 \mu \mathrm{m})$, and thick human hairs $(E=2.2 \mathrm{Gpa}$ for $\varnothing>80 \mu \mathrm{m}$ ), which occurs due to the different fraction of inner medulla.

It can be concluded that formaldehyde is not expected to significantly influence tensile results on hair. Differences of hair thickness may have some influence on $F_{\mathrm{E}}$, but deviations are within $2 \times$ errors even with the largest $\varnothing$ variability in human hair. It should be stressed that differences in $\varnothing$ do not transfer directly to differences in $F_{\mathrm{E}}$, as the elastic limit does not depend directly on $\alpha$. Average values in the rupture region are $F_{\mathrm{r}}=0.93 \pm 0.06$ $(\mathrm{CV} 31 \%) \mathrm{N}$ and $S_{\mathrm{r}}=0.57 \pm 0.03(\mathrm{CV} 26 \%)$.

\section{References}

Amaral LQ (1989) Early hominid physical evolution. Hum Evol 4:33-44 Amaral LQ (1996) Loss of body hair, bipedality and thermoregulation. Comments on recent papers in the journal of human evolution. . J Hum Evol 30:357-366

Astbury WT, Street A (1931) X-ray studies of the structure of hair, wool and related fibres. I.- General. Phil Trans Roy Soc London Ser A 230:75-101

Astbury WT, Woods HJ (1933) X-ray studies of the structure of hair, wool and related fibres. II.- The molecular structure and elastic properties of hair keratin. Phil Trans Roy Soc London Ser A 232:333-394

Bendit EG (1957) The alpha-beta transformation in keratin. Nature 179:535

Bowden FP, Tabor D (1956) Friction and Lubrication. In: Worsnop BL (ed) Methuen's monographs on physical subjects. Methuen, London, p 1967, (addendum)

Colombera KM, Joekes I (2004) Effect of hair conditioners on the mechanical and diffusion properties of hair, 15th Surfactants in Solution Symposium (SIS2004), June 6-11, Fortaleza, Brazil, Abstracts Pg. 70 (results from a Master Dissertation, University of Campinas, 16/04/2004, Brasil)

Darwin C (1871) The descent of man and selection in relation to sex. Murray, London

de Vore I (ed) (1965) Field studies of monkeys and apes. Holt, Rinehart \&Winston, New York

Doran DM (1997) Ontogeny of locomotion in mountain gorillas and chimpanzees. J Hum Evol 32:323-344

Etkin W (1954) Social behavior and the evolution of man's mental faculties. Am Nat 88:129-142

Feughelman M (1997) Mechanical properties and structures of alphakeratin fibres: wool, human hair and related fibres. UNSW Press, Sydney

Fossey D (1979) Development of the mountain gorilla: the first thirtysix months. In: Hamburg DA, McCown ER (eds) The great apes. Benjamin/Cummings, Menlo Park, California, pp 138-184

Fossey D (1983) Gorillas in the mist. Houghton Miffin, Boston

Franbourg A, Halergot P, Baltenneck F, Toutain C, Leroy F (2003) Current research on ethnic hair. J Am Acad Dermatol 48:S115-S119

Gennisson JL, Baldewek T, Tanter M, Catheline S, Fink M, Sandrin L, Cornillon C, Querleux B (2004) Assessment of elastic parameters of human skin using dynamic elastography. IEEE Trans Ultrason Ferroelectr Freq Control 51(8):980-989

Goodall van Lawick J (1967) Mother-offspring relationship in freeranging chimpanzees. In: Morris O (ed) Primate ethology. Aldine, Chicago, pp 287-346 
Gralen N (1952) Friction between single fibers. In: A discussion on friction. . Proc R Soc Lond Ser A 212:491-495

Gross J, Scherer GW, Alviso CT, Pekala RW (1997) Elastic properties of crosslinked resorcinol-formaldehyde gels and aerogels. J NonCryst Solids 211:132-142

Hoff MP, Nadler RD, Maple TL (1983) Maternal transport and infant motor development in a captive group of lowland gorillas. Primates 24(1):77-85

Honda I, Arai K, Mitomo H (1997) Characterization of cross-links introduced in gelatin. J Appl Polym Sci 64(10):1879-1892

Howell HG, Mieszkis KW, Tabor D (1959) Friction in Textiles. Butterworths, London

Iwamoto M (1985) Bipedalism of japanese monkeys and carrying models of hominization. In: Kondo S (ed) Primate Morphophysiology. Locomotor Analyses and Human Bipedalism. Tokyo University Press, Tokyo, pp 251-260

Jolly A (1972) The evolution of primate behavior. In: Simons EL, Pilbeam D (eds) The MacMillan series of physical anthropology. MacMillan, New York

Kingston JD, Marino BD, Hill A (1994) Isotopic evidence for neogene hominid paleoenvironment in the Kenya Rift valley. Science 264:955-959

Kittler R, Kayser M, Stoneking M (2003) Molecular evolution of Pediculus humanus and the origin of clothing. Curr Biol 13:1414-1417

Kleiber M (1975) The fire of life: an introduction to animal energetics, rev. edn. Pub Krieger RE, Malabar, Florida

Lockwood CA, Kimbel WH, Lynch JM (2004) Morphometrics and hominoid phylogeny: support for a chimpanzee-human clade and differentiation among great ape subspecies. Proc Natl Acad Sci USA 101:4356-4360

MacKinnon JR (1974) Behavior and ecology of wild orangutan. Anim Behav 22:3-74

Mahoney S (1980) Cost of locomotion and heat balance during rest and running from 0 to $55^{\circ} \mathrm{C}$ in a patas monkey. J Appl Physiol 49:789-800

Makinson KR (1952) Some comments on the paper by Dr. N. Gralen. In: A discussion on friction. Proc R Soc Lond Ser A 212:495-496

Montagna W (1982) The evolution of human skin. In: Chiarelli AB, Corrucini RS (eds) Advanced views in primate biology. Springer, Berlin, pp 35-41

Montagna W (1985) The evolution of human skin (?). J Hum Evol $14: 3-22$
Newman R (1970) Why man is such a sweaty and thirsty naked animal: a speculative review. Hum Biol 42:12-27

Nikiforidis G, Tsambaos D, Balas C, Bezerianos A (1993) A method for the determination of viscoelastic parameters of human hair in relation to its structure. Skin Pharmacol 6:32-37

Plooij FX (1984) The behavioral development of free-living chimpanzees babies and infants. In: Lipsitt LP (ed) Monographs on infancy. ABLEX, New Jersey

Potts R (1998) Environment hypotheses of hominin evolution. Yearbk Phys Anthropol 41:93-136

Richmond BG, Begun DR, Strait DS (2001) Origin of human bipedalism: the knuckle-walking Hypothesis Revisited. Yearbk Phys Anthropol 44:70-105

Robbins CR (1994) Chemical and physical behavior of human hair, 3 edn. Springer, New York

Rogers WR, Coelho AM Jr, Carey KD, Ivy JL, Shade RE, Easley SP (1992) Conditioned exercise method for use with nonhuman primates. Am J Primatol 27:215-224

Rogers AR, Iltis D, Wooding S (2004) Genetic variation at the $\mathrm{MC}_{\mathrm{I}} \mathrm{R}$ locus and the time since loss of human body hair. Curr Anthropol 45:105-108

Ruvolo M, Pan D, Zehr S, Goldberg T, Disotell TR (1994) Gene trees and hominoid phylogeny. Proc Natl Acad Sci (USA) 91:8900-8904

Schultz AH (1931) The density of hair in primates. Hum Biol 3:303-321

Schultz AH (1968) The recent hominoid primates. In: Washburn SL, Jay PC (eds) Perspectives on human evolution-1. Holt, Rimehart and Winston, New York, pp 122-195

Schwartz GG, Rosenblum LA (1981) Allometry of primate hair density and the evolution of human hairlessness. Am J Phys Anthropol 55:9-12

Silver FH (1987) Biological materials: structure, mechanical properties and modelling of soft tissues. New York University Press, New York

Tuttle RH, Watts DP (1985) The positional behavior and adaptive complexes of Pan Gorilla. In: Kondo S (ed) Primate morphophysiology, locomotor analyses and human bipedalism. Tokyo University Press, Tokyo, pp 261-288

Wheeler PE (1992) The influence of the loss of functional body hair on the water budgets of early hominids. J Hum Evol 23:379-388

Wolfram LJ (2003) Human hair: a unique physicochemical composite? J Am Acad Dermatol 48:S106-S114

Yunis JJ, Prakash O (1982) The origin of man: a chromosomal pictorial legacy. Science 215:1525-1529 